\title{
Data Analysis for Self-Driving Vehicles in Intelligent Transportation Systems
}

\author{
Hyunhee Park $\mathbb{D}^{1},{ }^{1}$ Kandaraj Piamrat, ${ }^{2}$ Kamal Singh, ${ }^{3}$ and Hsing-Chung Chen ${ }^{4,5}$ \\ ${ }^{1}$ Korean Bible University, Seoul, Republic of Korea \\ ${ }^{2}$ University of Nantes, Nantes, France \\ ${ }^{3}$ Laboratory Hubert Curien, University Jean Monnet, St-Etienne, France \\ ${ }^{4}$ Asia University, Taichung, Taiwan \\ ${ }^{5}$ China Medical University Hospital, China Medical University, Taiwan \\ Correspondence should be addressed to Hyunhee Park; parkhyunhee@gmail.com
}

Received 31 January 2020; Accepted 31 January 2020; Published 19 February 2020

Copyright (c) 2020 Hyunhee Park et al. This is an open access article distributed under the Creative Commons Attribution License, which permits unrestricted use, distribution, and reproduction in any medium, provided the original work is properly cited.

Self-driving vehicles are regarded as the future of transportation. In the near future, self-driving vehicles would ferry passengers from one place to another place, like driverless taxis, and transport packages and raw materials from city to city. However, for all the optimism surrounding self-driving vehicles, there is also an equal amount of scepticism and concern. Many people believe that self-driving vehicles will be "no safer" than human-controlled vehicles. Therefore, the willingness of the public to ride in a fully self-driving vehicle will be very low due to nonzero accident rates.

A lot more data and testing are required to influence the public's beliefs on self-driving vehicles being ready for the road. Collecting more datasets will help to improve self-driving car modelling using data analysis; however, an incremental approach has to be taken for in-depth exploration of data analysis techniques applied to self-driving vehicles. This is due to the lack of sufficient information regarding how rare traffic and weather events should be modelled in transportation systems.

This special issue aims to provide a comprehensive overview of the most recent and promising advancements of data analysis technologies for self-driving vehicles in intelligent transportation systems. Data analysis technologies for self-driving vehicles are expected to cover the current state of the art and highlight remaining challenges and barriers to the development of self-driving vehicles as part of intelligent transportation systems. 11 papers were submitted for this issue; 4 of them were accepted for publication.

Below, we provide an overview of the selected articles for this special issue.
Vehicle Movement Analyses Considering Altitude Based on Modified Digital Elevation Model and Spherical Bilinear Interpolation Model: Evidence from GPS-Equipped Taxi Data in Sanya, Zhengzhou, and Liaoyang. The modified digital elevation (MDE) model and spherical bilinear interpolation (SBI) model were proposed for vehicle movement analyses considering altitude. In addition, the experimental data of 9,990 GPS-enabled taxis in Sanya, Zhengzhou, and Liaoyang were adopted to support comparisons. Measurement results showed that the MDE model having over 99\% less disparity with direct solution as compared to the original model and SBI model could further improve the effects. In conclusion, the contributions of this study are as follows: (1) the MDE model was built to calculate vehicle movements by digital elevation data based on mathematical equations and (2) the SBI model was proposed and applied to improve the preciseness of GPS data with altitude of collaborative vehicles.

Malware Detection in Self-Driving Vehicles Using Machine Learning Algorithms. A machine learning-based data analysis method was proposed to accurately detect abnormal behaviours due to malware in large-scale network traffic in real time. First, the authors define a detection architecture, which is required by the intrusion detection module to detect and block malware attempting to affect the vehicle via a smartphone. Then, they propose an efficient algorithm for detecting malicious behaviours in a network environment and conduct experiments to verify algorithm accuracy and cost through comparisons with other algorithms. Here are the main contributions of this article: (1) the 
machine learning-based detection model was proposed for detecting adware and malware in a self-driving vehicle environment and (2) intrusion detection module architecture was defined to detect malware and prevent it from affecting the selfdriving vehicles through a smartphone.

Data Analysis for Emotion Classification Based on Bio-Information in Self-Driving Vehicles. To classify the electroencephalography (EEG) data into four types of biometric emotion information (stability, relaxation, tension, and excitement), a fuzzy control system was designed and the pulse rate and blood pressure data were composed in single packets separately and sent to the database. The authors propose a system for inferring emotion using EEG, pulse, and blood pressure (systolic and diastolic blood pressure) of the user and recommending color and music according to the emotional state of the user for a user service in self-driving vehicle.

An Improved Automatic Traffic Incident Detection Technique Using a Vehicle to Infrastructure Communication. An ITS model was proposed to estimate the traffic incident from a hybrid observer (HO) method and detect the traffic incident by using an improved automatic incident detection (AID) technique based on the lane-changing speed mechanism in the highway traffic environment. Moreover, the authors have further evaluated the performance of the proposed method with well-known techniques such as Naive Bayes, KNN, and SVM using I-880 traffic data. This study has the following contributions: (1) the authors developed the connection between vehicles and roadside units (RSUs) by using a beacon mechanism and (2) they utilized the probabilistic approach to collect the traffic information data, by using a vehicle to infrastructure (V2I) communication.

The guest editors of this special issue believe that the selected articles contribute in moving the self-driving vehicles field forward and open new avenues for further research of smart transportation. Especially, these 4 selected articles provide key ICT solutions to various challenges faced by the developers of technologies used for self-driving vehicle safety and intelligent transportation systems.

\section{Conflicts of Interest}

The guest editors declare that there are no conflicts of interest regarding this special issue.

\section{Acknowledgments}

The guest editors would like to thank the reviewers for the time and efforts devoted to the analysis of the submitted manuscripts, as well as for the comments and suggestions used to improve the quality of the accepted articles.

Hyunhee Park Kandaraj Piamrat Kamal Singh Hsing-Chung Chen 